\title{
ORIGEN, SIGNIFICADO Y PRÁCTICA DE LA “GOBERNANZA GLOBAL” EN LA ÉPOCA DE LA GLOBALIZACIÓN: UNA MIRADA HISTÓRICA DESDE LA PERSPECTIVA DE LOS CAMBIOS CLIMÁTICOS*
}

\author{
ORIGIN, MEANING AND PRACTICE OF "GLOBAL GOVERNANCE" IN THE ERA OF \\ GLOBALIZATION: A HISTORICAL VIEW FROM THE PERSPECTIVE OF CLIMATE CHANGE
}

\author{
Francesco Petrone ${ }^{1}$ \\ petrone.francesco@gmail.com \\ Universidad de Barcelona \\ Barcelona, España \\ DOI: https://doi.org/10.32735/S2735-61752019000116137
}

\begin{abstract}
RESUMEN
En este paper nos proponemos de contextualizar de manera crítica el origen y el sentido de la "gobernanza global" en la época de la globalización, y luego analizamos su aplicación en la gestión de los cambios climáticos. Antes de todo describimos el concepto de globalización, interpretado bajo su enfoque pluridisciplinar, subrajando su complejidad y sobre todo el efecto que ha habido sobre el policy making y la así llamada "crisis del Estado". Analizamos después el significato de la gobernanza global y sus implicaciones en una época en que ya no existe una centralidad en la gestión de problemas globales, si no que hay siempre más intereses diversos y plurales en juego. Finalmente aplicamos este concepto, bajo una perspectiva histórica, al ámbito de los cambios climáticos. Es éste un campo amplio, por eso nos limitamos a hablar, tomandolos como exemplos, de las cumbres originarias (Estocolmo y Río de Janeiro) y de la COP21 (Paris), para evaluar, finalmente, si y como es posible superar los límites de la gobernanza delante a semejantes desafíos.
\end{abstract}

Palabras claves: gobernanza, globalización, cambio climático, crisis del Estado, COP21.

\begin{abstract}
In this paper we contextualize, in a critical way, the origin and the meaning of "global governance" in the era of globalization. Then we analyze its application to the management of climate change. First of all we describe the concept of globalization, interpreted in its multidisciplinary approach, underlining its complexity and especially the effect that has had in
\end{abstract}

\footnotetext{
* Artículo recibido el 29 de septiembre de 2017; aceptado el 13 de diciembre de 2017.

${ }^{1}$ Parte del Liceo Scientifico G Peano. Doctor por Universidad de Barcelona: "Globalización, Gobernanza e Instituciones Internacionales". Mención: "Doctorado Internacional". Investigador invitado en el Instituto Ralph Bunche de Estudios Internacionales (Nueva York) y el Centro de Políticas BRICS (Río de Janeiro). Catedrático de Historia y Filosofía Mundial, investigador en "Gobernanza Global, Instituciones Internacionales y Potencias Emergentes". Profesor de Relaciones Internacionales en Geneva Business School (Barcelona y Astana).
} 
policy making and in the so-called "crisis of the State". Successively we analyze the meaning of global governance and its implications in a time when there is no longer a unique center in the management of global issues, but diverse and plural interests have grown. Finally, we apply this concept, from an historical perspective, to the field of climate change. This is a broad field, that's why we just talk about, taking them as examples, the original summits (Stockholm and Rio de Janeiro) and the COP21 (París), to evaluate, finally, if and how it is possible to overcome the limits of governance in the facing of such challenges.

Key words: Governance, globalization, climate change, state crisis, COP21.

"El primer objetivo de la gobernanza consiste en aprender a vivir juntos y administrar pacíficamente el hábitat común, asegurar en él las condiciones necesarias para la supervivencia, la paz, la plenitud y el equilibrio entre la humanidad y la biosfera".

Pierre Calame

\section{Introducción. Globalización y "crisis del Estado"}

En las últimas décadas, el escenario mundial ha sufrido transformaciones que han sacudido su estructura y generado cambios importantes. Históricamente, con la caída del Muro de Berlín (noviembre, 1989), se han mostrado nuevos escenarios que han dado al planeta un nuevo e incierto rostro. En primer lugar, después de 1989 las economías han pasado de ser casi puramente nacionales a convertirse en transnacionales: la apertura de las fronteras y el aumento de las relaciones comerciales han propiciado un incremento del comercio y una mayor interdependencia entre las economías del mundo.

Aunque históricamente el comercio haya sido cada vez más intenso entre los países, con la apertura de mercados en el Este estos intercambios se han vuelto más rápidos, dando lugar a lo que se ha definido como "Turboglobalización" (Mayos, 2011), es decir, una forma de globalización que se ha llevado a cabo con mayor rapidez e intensidad alterando el sistema económico internacional. Globalización es la palabra clave en los últimos años y está sujeta a muchos y continuos estudios e investigaciones. La globalización no describe una sola área de la vida social, sino que representa un cambio en varios aspectos de nuestras vidas y por ese motivo, para ser entendido, es necesario un enfoque multidisciplinario. De hecho, de acuerdo con un artículo del Foro de Política Global, las interpretaciones de la globalización son diferentes:

Para el economista, la globalización es esencialmente la creación de un mercado global. Para el historiador, es una época dominada por el capitalismo global. Para el sociólogo, la globalización hace hincapié tanto en la celebración de la diversidad como en la convergencia de las preferencias sociales en cuestiones de formas de vida y valores sociales. Para el politólogo, la globalización es la erosión gradual de la soberanía estatal. Mientras que el estudio específico sobre la globalización por parte de las diversas disciplinas desarrolla conocimientos ricos y particulares, cada una de 
ellas simplemente explica parte del fenómeno, tal y como ocurre en la proverbial descripción de un elefante por parte de seis hombres ciegos. Por esta razón, la globalización es más conocida como un concepto que trasciende las disciplinas individuales, pero al mismo tiempo las une. La globalización, por lo tanto, debe ser el resultado de un estudio de perspectiva multidisciplinar (Muqtedar, 2003).

Con el fin de comprender lo que está sucediendo a nivel político y social es fundamental, a nuestro entender, analizar el proceso de globalización. Éste, de hecho, ha tenido un impacto significativo en nuestras vidas y en las instituciones que históricamente han encarnado los puntos de referencia para las personas. Desde un punto de vista social, aunque no son pocos los autores que también ponen en evidencia los efectos beneficiosos de la globalización, lo que se puede comprobar con mayor claridad es que el mundo ha sufrido una división en dos categorías fundamentales, a saber, la de los "ganadores" y la de los "perdedores" de la globalización (Baumann, 1999). Estas dos categorías representan los dos polos creados como consecuencia de este estado de cosas: uno rico y detentor del poder generado por la globalización, es decir, aquéllos que sabían (y podían) beneficiarse de sus ventajas, mejorar su vida (Bauman, 1999, los llama "Turistas") y que representan una pequeña parte de la humanidad y, por otro lado, la gran mayoría de la población mundial que es, en cambio, una "víctima" de la globalización, es decir, se ha convertido en el "perdedor" porque ha sufrido los efectos en términos de flexibilidad laboral y precariedad de la existencia. En esta última categoría incluye a todos aquellos que se ven obligados a desplazarse donde hay más posibilidad de encontrar puestos de trabajo, que están perdiendo cada vez más derechos y protección social, y que, en general, son arrojados a un mundo dominado por el capitalismo salvaje (Bauman los define como "Vagabundos"). Para resumir este punto (y hacernos una idea de lo que estamos diciendo), las cifras indican que el $20 \%$ de la población mundial posee el $80 \%$ de la riqueza del planeta, mientras que el $80 \%$ de la población sobrevive dividiéndose el $20 \%$ de esas riquezas (Gallino, 2003). Estos datos describen "en síntesis" el escenario actual del mundo.

Según lo que ha declarado el pensador Thomas Pogge (2010): es cierto que ha habido muchos logros en la ciencia y en la tecnología, sin embargo, la brecha entre ricos y pobres no sólo se ha mantenido inalterable, sino que, ha aumentado. Este análisis es importante para entender lo que sucedió en los años ochenta y para introducir el tema central de nuestra discusión: la gobernanza mundial. Las consecuencias que se han registrado a nivel social y político han dado una nueva cara también al sistema de poder tal y como se había configurado en los años anteriores. De hecho, si inmediatamente después de la Segunda Guerra Mundial el papel del Estado-nación seguía siendo predominante, a partir del desarrollo de la globalización de las últimas décadas ha perdido gran parte de su dominio, tanto en cuestiones relativas a los asuntos internos de cada país, como a nivel internacional. Dentro de cada país el aumento de nuevas demandas sociales por parte de nuevos grupos que se hacen portavoces de nuevos intereses, han recibido respuestas inadecuadas por parte del propio Estado. Si bien históricamente los partidos políticos se habían convertido en los portavoces de las cuestiones sociales, con la apertura de las economías a nivel transnacional y con el aumento del activismo, la conciencia de nuevos grupos sociales, así como con el nacimiento de asociaciones, fundaciones, organizaciones no gubernamentales, el Estado ha encontrado muchas dificultades en la gestión de estas nuevas situaciones y de las demandas por parte de la sociedad. "Desde 
abajo" hemos asistido a un aumento real de participación y sensibilización que el Estado no ha sido capaz de hacer frente por sí solo, por estar poco preparado y carecer de los recursos necesarios para satisfacer estas demandas. La consecuencia ha sido que ha comenzado a delegar muchas de sus funciones a los nuevos actores sociales, tanto en el sector público como en el privado.

Si bien estos cambios tuvieron lugar en el ámbito estatal, incluso "hacia arriba" (bottom-up) se han presentado nuevos desafíos a los que los Estados no sabían ni podían responder de manera concreta, debido a sus pequeñas dimensiones comparadas con el contexto internacional en el que, por sí solos, no podían enfrentarse a los desafíos generados por la globalización. De hecho, a nivel internacional el nacimiento de instituciones regionales e internacionales ha sido, entre otros, el resultado de la necesidad de dar respuestas a problemas más grandes que superan las fronteras nacionales. En la práctica, los desafíos que plantea la globalización han provocado una erosión del Estado-nación, incluso hacia arriba, es decir, en la dirección de las instituciones transnacionales (véase, por ejemplo, la Unión Europea) a las que se les han delegado inevitablemente determinados aspectos de la soberanía que antes eran exclusivos del Estado-nación. Si tomamos como ejemplo la Unión Europea en el momento histórico en que vivimos, podemos ver que esta institución adolece de un déficit democrático que no consigue resolver y que socava sus cimientos, pero es innegable que las instituciones europeas desempeñan en Europa un papel importante y pueden llegar a influir en las políticas nacionales de muchos Estados. ¿Qué quiere decir esto? Mientras que esta "crisis del Estado" (Cassese, 2002) representa, por un lado, un cambio en la historia, significando la transición a un nuevo tipo de poder a nivel internacional, por el otro, es indicativo del hecho de que esta interdependencia es cada vez más fuerte, y entonces es necesario encontrar una manera de gestionarla buscando nuevas formas de distribución del poder que tengan en cuenta el nuevo escenario internacional que se ha ido creando. Este nuevo escenario se caracteriza por la presencia de actores tanto estatales como no estatales, tales como el Estado mismo, las organizaciones multinacionales, las asociaciones transnacionales, las organizaciones no gubernamentales, las fundaciones y otros actores (Rosenau, 2009), ${ }^{2}$ es decir, un "conjunto de participantes" (stakeholders) que están dando una nueva cara al poder que ya no es centralizado, sino que vive una fragmentación.

Este escenario se caracteriza entonces por una multiplicidad de intereses. La multiplicidad de los intereses deriva de la diversidad de actores que entran en juego en esta nueva forma de gestión del poder. En este escenario, es difícil hacer predicciones fiables sobre el futuro aunque hay algo, sin embargo, que parece evidente: no puede existir un mundo dominado por dos potencias (ahora superado por el fin del sistema bipolar de la Guerra Fría) ni un mundo unipolar donde solamente un país tiene el control del equilibrio internacional de poder, como en este momento de la historia hacen los Estados Unidos (que, en cualquier caso, ven su hegemonía cada vez más amenazada por la presencia de potencias emergentes, como el caso, a pesar de las recientes crisis, de los llamados países BRICS: Brasil, Rusia, India, China y Sudáfrica). Nos parece que en este contexto hay algo cierto: el modelo occidental (el de la democracia liberal de

\footnotetext{
2 J. Rosenau, Governance in the twenty-first century, in Whitman, J. (Editor), Global Governance, Palgrave MacMillan, New York, 2009. En este libro, el autor americano menciona los siguientes actores: las ONG, los movimientos sociales, las regiones (micro y macro) y los ayuntamientos (las ciudades), los Estados (que define como "issue regimes"), las asociaciones transnacionales, las agencias de rating, los tribunales interestatales o internacionales, la ONU y la Unión Europea, los mecanismos institucionales como los Observatorios electorales.
} 
tipo capitalista), que a finales de los años ochenta había declarado su victoria sobre el modelo socialista hasta el punto de que algunos autores habían declarado el "fin de la historia" (Fukuyama, 2003) con la victoria de la democracia, ya que esta representaba un optimum desde un punto de vista económico y de los derechos sociales, presenta ahora unos límites muy importantes que requieren una nueva manera de pensar acerca de las formas participativas a nivel político.

\section{Las críticas de Stiglitz y la "global governance"}

Joseph Stiglitz, economista estadounidense ganador del Premio Nobel en 2001, ex colaborador del gobierno de Estados Unidos (en calidad de presidente del Consejo de Asesores Económicos) y del Banco Mundial (como Vicepresidente Senior y Economista Jefe), ${ }^{3}$ ha señalado, en la que se considera como una de las criticas màs clasicas y apropiadas, como causa del "malestar en la globalización" (Stiglitz, 2002) las malas políticas que las instituciones internacionales como el Fondo Monetario Internacional (FMI), Banco Mundial (BM) y la Organización Mundial del Comercio (OMC) han utilizado en los últimos decenios del siglo pasado. En su opinión, las políticas de deuda que adoptan estas instituciones, y en particular las del FMI a las que Stiglitz dedica una amplia crítica (y que acusa de haber impulsado reformas devastadoras para la mayor parte de los países del mundo. Reformas en estilo neoliberal, como, sobre todo: flexibilización de los mercados, abertura a inversiones extranjeras y privatizaciones) han creado desequilibrios en detrimento de los países que ya se encuentran en desventaja, los del llamado "Tercer Mundo", en favor de los países occidentales (y en particular las grandes multinacionales de éstos) que han tomado ventaja con esta política al crear una creciente dependencia de los países del Tercer Mundo respecto a ellos.

A raíz de esta situación global tan caótica, en los últimos años se han ido pensando diferentes maneras, teóricamente más adecuadas a esa realidad fragmentada, de enfrentarse a estos grandes conflictos. En particular, ha ido tomando forma la que se quiere proponer como una posible "respuesta" a esta situación: la llamada "Gobernanza Global" (Global Governance).

Este término indica, en general, un proceso que la Comisión sobre Gobernanza Global en 1995 define de la siguiente manera:

La suma de las muchas maneras en que los individuos y las instituciones, públicas y privadas, manejan los asuntos comunes. Es un proceso continuo a través del cual los conflictos y los distintos intereses pueden conciliarse y pueden iniciar una acción cooperativa (Commission on Global Governance, 1995). ${ }^{4}$

Este concepto indica un proceso que no está definido pero que da lugar a nuevas y muchas

\footnotetext{
${ }^{3}$ Fuente: www.josephstiglitz.com

${ }^{4}$ Commission on Global Governance, Our Global Neighbourhood, Oxford, Oxford University Press, 1995. Texto original: "The sum of many ways individuals and institutions, public and private, manage their common affairs. It is a continuing process through which conflicting or diverse interests may be accommodated and co-operative action taken. It includes formal Institutions and regimes empowered to enforce compliance, as well as informal arrangements and institutions that people either have agreed to or perceive to be in their interest" (p. 70).
} 
investigaciones: si se escribe la palabra "gobernanza" en un motor de búsqueda, ¡se obtienen más de treinta y cinco mil resultados! (Weiss, 2013).

Aunque el concepto de gobernanza global ha tenido definiciones contrastantes, creemos que es clave para comprender las relaciones que se dan en la toma de decisiones en la actualidad: hay muchos actores involucrados en el proceso de toma de decisiones, porque aumentan las necesidades y demandas sociales a las que se les piden respuestas comunes. La gobernanza global en teoría expresa la necesidad de tener en cuenta el hecho de que hay diferentes puntos de vista y necesidades que deben ser valoradas y consideradas en un proceso de gestión política. En resumen, la democracia tradicional está siendo reemplazada por esta nueva forma de "hacer política" (policy making en inglés), que promueve una mayor participación y reclama el derecho a la heterogeneidad, a diferencia de la vieja forma de hacer política, que, con el tiempo, ha demostrado ser insuficiente para responder a problemas comunes y diferentes al mismo tiempo. Por lo tanto, la gobernanza entendida así, y teniendo en cuenta muchas de las limitaciones que la caracterizan y le dan un carácter ambiguo, quiere ser la expresión de una respuesta, en el sentido de una forma diferente de gestión, a estos problemas que tenemos en común.

Sin embargo, la idea que resulta, tras analizar gran cantidad de escritos sobre esta temática, es que la gobernanza no representa, de hecho, un aumento de la participación real, sino que esconde muchas limitaciones que dan como resultado, paradójicamente, una exclusión en la toma de decisiones y del policy making. De hecho, aunque durante estos procesos se tienen en cuenta los problemas comunes, en la práctica, cuando se toman decisiones que luego afectan a todos, solamente se atiende a la importancia económica y a la influencia política de las partes que participan al proceso de toma de decisión (los stakeholders). En este sentido, entre los diversos autores que han criticado este funcionamiento de la gobernanza, nos parece sugestivo mencionar el texto de Sandra Kroeger (2008) que analiza los procesos de participación de la sociedad civil en la Unión Europea y describe cómo muchas asociaciones, organizaciones no gubernamentales o grupos de interés -que no tienen el mismo poder económico que las empresas multinacionales, por ejemplo-, son considerados sólo durante la fase de consulta de la discusión de un problema particular. Luego, en cambio, en el momento de la decisión final, la opinión de estos grupos pesa poco y su aporte a la toma de decisión se agota, suplantada por el poder económico de los que tienen más peso para influir en los resultados de las decisiones sobre ciertos temas (ver los casos de los poderosos lobby en Bruselas, que tienen un poder casi incontestable). De aquí, por supuesto, el título de su trabajo: "Nothing but consultation", es decir, "nada más que consultores", y no protagonistas de las decisiones políticas.

Por tanto, es innegable que, de acuerdo con estas primeras etapas, la gobernanza tiene algunos puntos poco claros y aún está lejos de ser considerada como una "nueva forma de hacer política", porque una vez más parece que viejas formas de hegemonía se presentan bajo el disfraz de un nuevo tipo de participación política que, en la práctica, está muy lejos de lo que se propone en la teoría. Sin embargo, a pesar de estas limitaciones es innegable que la gobernanza describe una situación en la que, de hecho, se encuentra el mundo: la de una gran interdependencia y un momento histórico en el que se necesita una participación más amplia en las cuestiones políticas. Al mismo tiempo, como causa de la explotación del planeta, las guerras y otras cuestiones que se han generado con esa globalización sin control, el planeta se enfrenta ahora a cuestiones de importancia vital para su propia supervivencia. Hoy en día problemas como el cambio climático, la pobreza, el terrorismo internacional, las crisis financieras, las grandes migraciones, las organizaciones criminales, la destrucción del medio ambiente, las 
violaciones a los derechos humanos y así sucesivamente, plantean decisiones cruciales que necesitan respuestas colectivas e inmediatas (AA.VV., 2012). De acuerdo con las estadísticas y los estudios de caso (AA.VV., 2012), que ahora son numerosos, hay muchos peligros debido a que los recursos se están agotando, la tierra de cultivo se desgasta cada vez más, la población mundial aumenta y el cambio climático está destruyendo el planeta.

Siendo así, nos preguntamos si una "gobernanza humana" (Falk, 1995) es posible. Es decir, una gobernanza que tenga en cuenta estas necesidades tan urgentes que pertenecen a todo ser humano y que tengan en cuenta la importancia de su defensa en todos ámbitos.

Hay muchos problemas que afectan a todas y todos. Sin embargo, para dar un ejemplo concreto, cercano y que nos parece entre los más urgentes en la actualidad, aquí tomamos en cuenta el ejemplo de los cambios climáticos. Analizando la manera en que la gobernanza global ha funcionado y funciona en este ámbito, pretendemos dar un ejemplo a raíz del cual verificar si es posible hablar de una "gobernanza que funcione", es decir que tenga en mente su función de dar respuestas a problemas de interés general.

\section{Gobernanza local y cambio climático}

La situación de constante crisis global actual, en la que parece difícil encontrar compromisos y soluciones adecuadas, viene definida como "Gridlock" (literalmente: embotellamiento) por David Held (Hale, Held e Young, 2013). El Gridlock describe una situación de "paro" a nivel mundial, en la que no se sabe bien qué dirección tomar para enfrentar problemas globales, como en el caso del cambio climático. Sin embargo, hay algunas visiones que intentan buscar una salida de este impasse. Según estas visiones, para salir del Gridlock hay que entender la naturaleza de la gobernanza. Sin embargo esa naturaleza tiene interpretaciones, contrastantes, que vamos a explicar aquí para tener una visión "dialéctica".

La primera, por parte de Friedrich, cree que se trata de un proyecto neoliberal que ha sido organizado por la sociedad civil transatlántica para establecer una nueva forma de hegemonía (anglosajona) a nivel internacional (Friedrichs, 2009). La segunda es la de Thomas Weiss (2013): la global governance es un proceso en devenir que tiene que superar unos límites (gaps) para que pueda funcionar de cara al futuro. Está claro que ambas visiones tienen su parte de razón, sin embargo, lo que nos interesa aquí es procurar entender si la gobernanza puede dar una respuesta clara a los problemas globales.

¿Podemos pensar que los gaps que indica Weiss pueden ser superados? ¿O, como nos dice Friedrich, es un proceso que presenta aparentemente cambios, pero en la práctica no es más que la "vuelta al ataque", como podríamos denominarla, de los grupos económicos y políticos dominantes?

En el año 2015 ha habido eventos a partir de los cuales se ha querido plantear formas aparentemente nuevas de acción sobre diferentes asuntos globales como la pobreza, el cambio climático, las enfermedades etc. Por ejemplo, el 2015 fue el año en que se terminaba el plazo para alcanzar los MdG (Objetivos del Milenio de las Naciones Unidas). Pero, a pesar de las esperanzas optimistas iniciales, quedaba aún mucho por hacer. Por eso se han creado los SdG -en español, Objetivos de Desarrollo Sostenible (OdS)- a alcanzar para el 2030. Pero en particular el 2015 ha sido el año en el que se ha intentado dar el impulso más fuerte a los problemas relativos al cambio climático y al medioambiente. 
Sin embargo, antes de llegar a la cumbre de 2015 en París (COP21), ha habido circunstancias en el pasado en que se han realizado intentos por dar respuestas al problema climático. Se ha intentado construir una mayor participación y una gobernanza que funcione, pero aún falta mucho por hacer y cada día se lanzan nuevas alarmas.

Siguiendo un orden que incluye sólo las cumbres que nos parecen más significativas (incluirlas todas sería imposible), podemos intentar ver cuál ha sido la evolución de estos procesos relativos a las políticas del cambio climático hasta la COP 21 en París. En noviembre de 2016 se realizó otra cumbre en Marruecos (Marrackech - COP22) en la que no se realizaron avances importantes después de París, sino que, se ha vuelto a reiterar el mantener las promesas hechas durante la COP21. Y lo mismo se puede decir de la COP23 de 2017 en Bonn, Alemania.

A continuación, examinamos las cumbres que históricamente nos han parecido más relevantes. No incluimos Kioto y Copenhague -aunque sería también importante hacerlo-, por motivos de espacio, y porqué nos parece que las tres cumbres que vamos a analizar son más sugerentes para comprender cómo ha funcionado la gobernanza global, sobre todo bajo la perspectiva de "despertar" y crear conciencia sobre el problema.

\section{Conferencia de las Naciones Unidas en Estocolmo 1972}

En 1972, se convocó en Estocolmo la primera conferencia sobre medioambiente. Esta conferencia es importante porque representa el primer intento de enfrentar los problemas relativos al medioambiente. En particular, la Carta de las naciones Unidas no hacía referencia específica a la defensa del ambiente, pero sí, en el preámbulo, se hace mención al desarrollo social y la mejora de los estándares de vida. Así es que la conferencia fue una consecuencia de las primeras sensibilidades hacia esta temática, sobre todo por parte del mundo científico, despertadas por la actividad humana en el planeta, en concreto debido a la producción industrial y otras actividades como los experimentos nucleares.

La cumbre de Estocolmo representa el primer intento de sensibilizar sobre el problema del medioambiente y de las consecuencias que una actividad explotadora puede haber sobre ello.

Sin embargo, la situación política a nivel internacional tenía características bien particulares. En 1972 había muchos problemas de fragmentación geopolítica, debido a nuevos países independientes y a su voluntad de mantener su soberanía sobre cuestiones internas. Una coordinación global fue complicada porque resultó difícil coordinar intereses distintos y hacerlos converger hacia decisiones vinculantes, es decir decisiones que conllevasen un compromiso verdadero y concreto, obligando a su cumplimiento, sobre todo vinculado a un acuerdo de tipo global con otros países, hacia un desarrollo sustentable respetando el medioambiente.

Sin embargo, el punto que se puede destacar más sobre esta cumbre fue el nacimiento de una agencia de la ONU, la UNEP (United Nation Environment Programme). Aunque esta agencia representó seguramente un logro importante y una sensibilidad hacia estos problemas, pronto se reveló débil debido a la importante fragmentación de intereses entre los países que participaron a la cumbre. Por lo que esta experiencia, pese a que intentó llamar la atención sobre el problema, por otra parte reveló su fragilidad debido a esta fragmentación, y al mismo tiempo a los problemas creados por varias organizaciones ambientales que no querían ceder su 
autoridad, y recursos, a otras organizaciones.

A nuestro parecer, esta conferencia tuvo otros logros, a saber:

1) A pesar de esta fragmentación, en Estocolmo se logró poner el problema del medio ambiente y del clima en el centro del debate y como uno de los problemas principales de las agendas globales. En la Declaración final se acordaron 26 principios sobre los derechos y las responsabilidades humanas sobre el medioambiente. Estos principios fueron fundamento para otras cumbres y acuerdos siguientes, y al mismo tiempo, son la base a partir de la cual se ha desarrollado el derecho internacional a este respecto.

2) Además, se crearon coaliciones para poner en marcha nuevas medidas para contrarrestar la polución. Por ejemplo 1) el "grupo de Toronto", en 1983, que abogaba por la prohibición de los Clorofluorocarburos (CFC), esto es, unos derivados de los hidrocarburos; y 2) El "protocolo de Montreal", de 1987, que preveía la disminución y/o eliminación de sustancias que dañan la capa de ozono.

3) Publicación de "Our Common Future": en 1983 la Asamblea General de la ONU creó el WCED (World Commission on Environment and Development) para "proponer estrategias a largo plazo para alcanzar desarrollo sostenible para el 2000". La Comisión publicó en 1987 el reporte: Our Common Future. Según este reporte el desarrollo sostenible se tiene que basar en los siguientes puntos fundamentales: crecimiento económico, protección ambiental e igualdad social.

\section{Río de Janeiro 1992}

La cumbre de Río de Janeiro en 1992, representa, a nuestro entender, otro momento interesante para analizar la situación relativa a los progresos sobre el cambio climático.

Sin embargo, a pesar del optimismo inicial, en Río de Janeiro tampoco se alcanzaron resultados decisivos. En efecto, hubo diferentes factores que influyeron en el desarrollo de la cumbre: como en 1972 se evidenciaron varios problemas como una división entre países (Norte-Sur), la poca voluntad de ceder soberanía en temas ambientales y una falta de coordinación: los EE.UU. rechazaron la propuesta de países europeos, respecto de lograr un compromiso sobre el cambio climático, asunto que influyó la decisión de otros países en cuanto a no tomar medidas contra este problema. En Río de Janeiro no se alcanzaron resultados en el ámbito climático.

Pero como en Estocolmo, fue importante llamar la atención sobre los problemas ambientales. En este contexto, se fueron preparando las bases del futuro protocolo de Kioto (con sus límites) y la movilización a nivel mundial aumentó considerablemente.

La cumbre de 1992 representa uno de los más grandes intentos de enfrentarse al problema. Entre otros, participaron alrededor de 25 mil ONG y muchos representantes de la sociedad civil. El objetivo, en breve, era crear un compromiso en el que los países desarrollados hubieran asistido a los países en vías de desarrollo, económicamente y en tecnología, para lograr resultados en ámbitos medioambientales como los bosques y la atmósfera y crear un consenso para actuar conjuntamente contra las actividades que dañan el mundo (Held, Nag and Roger, 2013). Aunque los logros en este sentido fueron escasos, es preciso destacar cómo se empezó a dar vida a una gobernanza global más concreta, por ejemplo, por el mismo hecho de extender 
la participación a un número mayor de miembros de la sociedad civil, que en teoría se hicieron portavoces de demandas por parte de sectores de la población mundial que, en mayor o menor medida, son los afectados por los cambios climáticos.

\section{COP 21 París, 2015: logros y límites}

La Conferencia de las Partes de 2015 en París (COP21), fue presentada como el momento decisivo para tomar medidas sobre el cambio climático, cada día más preocupante, a causa de todas las manifestaciones desastrosas que hubo en los últimos tiempos: terremotos, huracanes, deshielo de los glaciares, inundaciones, temperaturas anormales, etc. Se presentaba un escenario catastrófico al que se tenía que dar respuestas globales rápidas y concretas: es decir la reducción del calentamiento global que no tiene que superar los 2 grados centígrados. Durante la conferencia, se propuso bajar a 1,5 grados este aumento de las temperaturas. Sin embargo, esta petición, que fue propuesta por los países más afectados por el problema, encontró mucha resistencia por parte de los países más industrializados.

De todas formas, para lograr el objetivo de contener el calentamiento global, se tienen que empezar a reducir las emisiones globales. Sin embargo, a pesar de las declaraciones tan inquietantes, y después que se definieron como "históricos" los logros de los acuerdos de París, los resultados finales presentaban unos límites importantes.

En primer lugar, el acuerdo no es vinculante en el sentido de que, si bien vincula a alcanzar la reducción del calentamiento bajo los 2 grados, no obliga a los estados en relación a las medidas a tomar para las reducciones de las emisiones. Además, no prevé sanciones por parte de quien no respeta los acuerdos de reducción de las emisiones. Esto significa que los países que participaron en la cumbre pueden ratificar el acuerdo, pero prácticamente mantienen las emisiones en base a sus propias exigencias. Por ejemplo, algunos países pueden actuar, para reducir las emisiones que producen, siguiendo tiempos distintos, en base a sus necesidades, debido a "su reciente industrialización". Se ha hablado en este caso de "responsabilidad no diferenciada".

El texto final preveía que los acuerdos fueran efectivos en el momento en que se fuesen ratificados por lo menos por parte de 55 países (o por un número de países que produce el equivalente de $55 \%$ de las emisiones globales). Esto fue alcanzado, pero la reducción de las emisiones debe comenzar a partir de 2020, es decir en unos años más: un hecho incoherente, si se piensa que el problema del cambio climático se encuentra ya en un estado muy avanzado. Otro aspecto es que se habrá una revisión de los resultados alcanzados cada cinco años. Habrá una revisión previa en 2018, pero tendrá como objetivo prepararse para el 2020, y después será cada cinco años. ¡Es decir que la primera revisión sobre cómo se está actuando será en 2023!

En la COP 21 no se ha previsto ninguna cláusula específica sobre respeto y protección de los derechos humanos (EE. UU. y la Unión Europea tardaron un día más negociando por ese motivo). Y, otro aspecto muy importante, es que no se ha expresado ninguna voluntad, y tan poco propuesto ninguna fecha límite, de disminuir (con el objetivo de terminar) la explotación de gas, carbón y petróleo (Neutralidad Climática) y remplazar estas fuentes de energía con energías renovables. Al mismo tiempo no hubo ningún objetivo o propuesta para alcanzar la Deforestación Cero. 
En este escenario, si bien se ha intentado dar realce al compromiso tentativo por parte de los participantes, son muy grandes los límites de la gobernanza global de cara a los 196 países que participaron. Al mismo tiempo, se ha evidenciado una vez más, cómo en esta cumbre también se ha registrado una fuerte influencia de grandes grupos empresariales y de lobbies como los del petróleo, de los transportes (por ejemplo, tampoco se ha intentado procurar alguna medida sobre la reducción de las emisiones por parte de los aviones), etc. Esto es, los "ganadores" de la globalización.

No obstante que la COP21 tenía que ser una cumbre verdaderamente importante, y que debía dar paso a otra manera de enfrentar el problema, se ha llegado a la conclusión, que muy pocos objetivos previstos se han alcanzado. Y, a raíz de todo eso, es preciso también recordar que hay consecuencias importantes y muy impactantes que el cambio climático tendrá sobre las próximas generaciones. Sobre todas ellas: la ONU prevé 250 millones de personas que van a desplazarse de sus países de origen para 2050 (la mayoría por cuestiones relativas a cambios climáticos), generando un flujo migratorio muy grande y con todas las consecuencias económicas, sociales y medioambientales que ello conlleva.

Otro punto que no se ha tenido en cuenta durante la COP 21, es que muchos países más expuestos a los problemas del cambio climático pedían un acuerdo más "solidario", es decir, que tomara en cuenta también las necesidades de países más pequeños y más afectados por los efectos. Pero: ¿Qué se podía hacer durante la COP21?

A pesar de todo, los objetivos que se podían alcanzar eran claros, y un compromiso tenía que contemplar estas necesidades más concretas para realmente poner freno al calentamiento global. Entre estas medidas que se podrían haber tomado, se debía: comprometerse de manera verdaderamente vinculante a reducir en 1,5 grados las emisiones globales de CO2 antes de final del siglo; invertir 100 billones por año en países, con más riesgo de ser afectados por las consecuencias del cambio climático, donde es más urgente implementar "mitigación y adaptación" (mitigar se refiere a los instrumentos y a las prácticas concretas para reducir las emisiones; mientras que la adaptación se refiere, por otro lado, a invertir para reaccionar a las consecuencias que se han generado hasta ahora por motivo del cambio climático); otro punto importante, y que no ha tenido mucha resonancia durante la cumbre, ha sido el problema de la indemnización a los países que han resultado más afectados por los cambios climáticos producidos por los países Occidentales más industrializados. No se ha avanzado en ninguna propuesta de indemnizar a quien ha pagado caras las consecuencias de los cambios climáticos generados por parte de los países más desarrollados económicamente.

\section{Conclusiones}

Las críticas de otro pensador como Pierre Bordieu (1998), tienen en común con las de Stiglitz (2002), la idea de que se puede hablar, con todo derecho, de una globalización neoliberal decidida desde arriba, es decir de parte de los países más poderosos (o sea los ganadores de la segunda guerra mundial). En este escenario, un papel fundamental ha sido desempeñado por las instituciones internacionales creadas y encargadas de la gestión de este proceso. Entre éstas, recordemos, ha jugado un papel importante, según Joseph Stiglitz, el FMI. Con la aplicación de las políticas de deuda (es decir los prestamos que se daban a los países en crisis y que al fin y al cabo generaban dependencia de esta institución ya que esos préstamos se debían devolver con grandes intereses), que fueron erróneamente definidas como "buena gobernanza" (Hermet, 2008), el FMI ha creado un sistema de dependencia de los países en 
desarrollo hacia los occidentales (principalmente EE.UU.). Sin embargo, los problemas no sólo están relacionados con aspectos económicos. En efecto, como otros autores han descrito, a nivel subjetivo y antropológico (Castoriadis, 2006; Latouche, 2007), este sistema ha generado seres humanos pasivos y consumidores de los productos que produce.

Así que, para concluir este punto, tanto en términos de política internacional como en el ámbito nacional, los efectos negativos de la globalización son evidentes y la creciente brecha económica entre ricos y pobres es uno de los ejemplos.

Ahora bien, hemos visto como delante de problemas tan importantes y que afectan a todo el planeta como el cambio climático, se tiene dificultad para encontrar compromisos que sean verdaderamente vinculantes, esto significa que tengan obligaciones claras y concretas, y puedan dar reales respuestas globales a problemas que son globales. La dificultad principal reside sobre todo en que cada país sigue teniendo objetivos de interés nacional, como es normal, que representan un obstaculo, en los cambios climáticos pero también en otros asuntos globales. Al mismo tiempo la práctica actual de la gobernanza global nos ha hecho entender que falta mucho, o quizás es algo imposible a realizar, para construir un sistema en el que se cumpla con una una participación más amplia y que tenga en cuenta de prioridades comunes. Aún está lejos, o quizás sea una utopía, una gobernanza global que verdaderamente quiera ser efectiva y concreta.

Los límites evidenciados por Weiss nos hacen entender que también en la busqueda de soluciones más rápidas a los gaps, todo se reduce al business as usual, excluyendo aquellas iniciativas, muchas veces "desde abajo" de parte de la sociedad civil, que intentan interferir contra este statu quo.

De cara al futuro, y teniendo en cuenta cómo en los últimos tiempos los problemas globales, han ido in crescendo, se vuelve esencial también buscar un sentido más claro y más práctico a la función de la gobernanza global. Para que funcione, la gobernanza global debe considerarse como un medio por el que, confluyendo los intereses más diversos, las decisiones tomadas se hagan más efectivas. En la práctica, como nos dice Pierre Calame, la gobernanza nos tiene que enseñar "a vivir juntos y administrar pacíficamente el hábitat común, asegurar en él las condiciones necesarias para la supervivencia, la paz, la plenitud y el equilibrio entre la humanidad y la biosfera". 


\section{Referencias}

AA.VV. (2012). Le monde n'a plus de temps à perdre. Appel pour une gouvernance mondiale et responsable. Paris: éditions Les Liens qui Libèrent.

AA.VV. (2012). Où va le monde? 2012-2022 : une décennie au devant des catastrophes. Paris: Fayard.

Bauman, Z. (1999). Dentro la globalizzazione. Le conseguenze sulle persone. Roma-Bari: Editori Laterza.

Bourdieu, P. (1998). Contre-feux. Propos pour servir à la résistance contre l'invasion néolibérale. Paris: Editions Liber.

Cassese, S. (2002). La crisi dello Stato. Roma-Bari: Edizioni Laterza.

Castoriadis, C. et al. (2006). Una Sociedad a La Deriva: Entrevistas y Debates (1974- 1997). Buenos Aires: Katz Print.

Commission on Global Governance. (1995). Our Global Neighbourhood. Oxford: Oxford University Press.

Falk, R. (1995). On Human Governance. Toward a New Global Politics. Cambridge: Polity Press.

Fukuyama, F. (2003). La fine della Storia e l'ultimo uomo. Milano: Rizzoli.

Friedrichs, J. (2009). Global governance as liberal hegemony, in Jim Whitman (Ed.), Global Governance. Basingstoke: Palgrave Macmillan, 105-122.

Gallino, L. (2003). Globalizzazione e disuguaglianze. Roma-Bari: Editori Laterza.

Hale, T.; Held, D. \& Young, K. (2013). Gridlock: why global cooperation is failing when we need it most. Cambridge: Polity Press.

Held, D.; Roger, C. \& Nag, E. M. (2013). Climate governance in the developing world. Cambridge: Polity Press.

Hermet, G. (2008). Populismo, democracia y buena gobernanza. Barcelona: EI Viejo Topo.

Kroger, S. (2008). Nothing but consultation: The place of organized civil society in EU policymaking across policies. European Governance Papers, No. C-08-03.

Latouche, S. (2007). Sobrevivir Al Desarrollo: De La Descolonización Del Imaginario Económico a La Construcción De Una Sociedad Alternativa. Barcelona: Icaria.

Mayos, G. (2011). Aspectos nueva globalización. Primsa Social-Nuevas Formas de Relación Social, 6.

Muqtedar, K. (2003). The Globalist, agosto 28. Recuperado de: www.globalpolicy.org

Pogge, T. (2010). Povertà mondiale e i diritti umani. Responsabilità e riforme cosmopolite, Roma-Bari: Laterza editori.

Rosenau, J. (2009). Governance in the twenty-first century, in Whitman, J. (Ed). Global Governance. New York: Palgrave MacMillan.

Stiglitz, J. (2002). La globalizzazione e i suoi oppositori. Torino: Einaudi.

Weiss, T. G. (2013). Global Governance: Why? What? Whither? Cambridge: Polity Press.

Zolo, D. (2004). Globalizzazione, una mappa dei problema. Roma-Bari: Editori Laterza. 
$\sim 68 \sim$ 
